\title{
Mutational analysis of fructose-1,6-bis-phosphatase FBP1 indicates partially independent functions in gluconeogenesis and sensitivity to genotoxic stress
}

\author{
Ali Ghanem, Ana Kitanovic, Jinda Holzwarth, Stefan Wölfl* \\ Institute of Pharmacy and Molecular Biotechnology, Heidelberg University, Heidelberg, Germany. \\ * Corresponding Author: \\ Stefan Wölfl, E-mail: wolfl@uni-hd.de
}

\begin{abstract}
Fructose-1,6-bisphosphatase (FBP1) is a key enzyme in the evolutionary conserved pathway of gluconeogenesis. We had shown in an earlier study that FBP1 is involved in the response and sensitivity to methylmethanesulfonate (MMS)-induced DNA damage in yeast. In the work presented here we performed an alanine screen mutational analysis of several evolutionary conserved amino acid residues of FBP1, which were selected based on conserved residues and structural studies of mammalian and yeast homologues of FBP1. Mutants were examined for enzymatic activity, and yeast cells expressing these mutants were tested for growth on nonfermentable and MMS-containing media. The results obtained support predicted vital roles of several residues for enzymatic activity and led to the identification of residues indispensable for the MMS-sensitizing effect. Despite an overlap between these two properties, careful analysis revealed two mutations, Asn75 and His324, which decouple the enzymatic activity and the MMSsensitizing effect, indicating two distinctive biological activities linked in this key gluconeogenesis enzyme.
\end{abstract}

\author{
doi: $10.15698 /$ mic2017.02.557 \\ Received originally: 19.04.2016; \\ in revised form: 01.01.2017, \\ Accepted 17.01.2017, \\ Published 01.02.2017.
}

Keywords: FBP1, S. cerevisiae, MMS, genotoxicity, DNA-damage,

gluconeogenesis.

\section{Abbreviations:}

MMS - methyl-methanesulfonate, wt-wild type.

\section{INTRODUCTION}

Fructose 1,6 bisphosphatase (FBP1) has been known as the key-enzyme for gluconeogenesis, it irreversibly mediates the splitting of fructose 1,6 bis-phosphate into fructose 6 phosphate and inorganic phosphate. While gluconeogenesis shares most of its enzymes with glycolysis, Fbp1p is the only distinctive gluconeogenesis enzyme in the upper part of the gluconeogenesis pathway, through which the flux of this pathway can be regulated.

Gluconeogenesis has been thoroughly investigated under normal physiological conditions and in the adaptation to different nutrient conditions, and its importance in securing vital glucose homeostasis has been understood. However, the role of gluconeogenesis regulation and its effects in other conditions as for example during the development of tumours have long been overlooked [1].

The preference towards aerobic glycolysis as a major energy supply source is a common metabolic hallmark of both cancerous cells and many unicellular organisms including the yeast $S$. cerevisiae, both being aerobic fermenters with the major fermentation product being lactate in cancerous cells and ethanol in S. cerevisiae [2-4].

The induced glycolysis in cancers known as 'The Warburg Effect', its underpinnings and advantages for tumours form the cornerstone of our comprehension of cancer metabolism; nevertheless several additional aspects of tumour metabolism have remained in the shadow for decades [5]. In the last few years, a decisive role of FBP1repression in the initiation of several tumour types has been described; metabolically, the loss of FBP1 increased glycolytic flux, and decreased respiration [6-8]; morphologically, FBP1-loss has been demonstrated to be essential for the epithelial to mesenchymal transition (EMT), an essential morphological reprogramming step of epithelial tumor cells required for the dissemination of tumor cells from primary tumors, subsequently leading to the formation of metastasis [7].

Aerobic fermentation is also a hallmark of the yeast $S$. cerevisiae when grown in glucose rich medium and tightly controlled by nutrient sensing mechanisms $[9,10]$. Others and we have shown that there is a close link between metabolism and sensitivity to DNA damage in yeast cells [11, 12].

We had shown that expression of $F B P 1$ is induced in response to treatment with the genotoxic agent methylmethanesulfonate (MMS) and that sensitivity of the yeast $S$. cerevisiae to MMS treatment is correlated with FBP1 expression [13]. High levels of Fbp1p obtained by overex- 
pression increased sensitivity, while lack of FBP1 reduced sensitivity to MMS, which suggests a regulatory role for this enzyme in DNA damage response and in the resulting oxidative stress conditions [13]. This multi-faceted impact in addition to the high evolutionary conservation of several regulatory mechanisms and their related functions between the yeast and mammalian homologues, make yeast an attractive model-system to further probe and understand the multiple effects of $F B P 1$. Here we present and discuss the results from a mutational screening of several evolutionary conserved amino acid residues of Fbp1p, predicted to be important for catalysis and/or allosteric regulation of this enzyme. We assessed the impact of these mutations on enzymatic activity, and further investigated their effect for growth on non-fermentable medium and sensitivity of yeast cells harbouring these mutant enzymes towards MMS treatment. In our study, we identified several indispensable residues for catalysis and MMS sensitization. The results obtained range from complete inactivation of the enzymatic activity of Fbp1p to partially deactivating mutations. Moreover, two mutations have been found, Asn75 and His325, which decouple catalysis from MMS-sensitization.

\section{RESULTS}

Selection of amino acid residues for mutational analysis We prepared a small library of mutated yeast $f b p 1$ expression cassettes, each with a single point mutation, changing one evolutionary-conserved residue. Global alignment of the amino acid sequence of Fbp1p of Saccharomyces cerevisiae against that of human Homo sapiens, pig Sus scrofa, mouse Mus musculus and another yeast Candida albicans

CLUSTAL O(1.2.1) multiple sequence alignment



47

47

47

57

107

107

107

115

116

167

167

167

174

176

227

227

227

234

236

285

285

285

281

296

FIGURE 1: Multiple global-alignment of FBP1 amino-acid sequences of Saccharomyces cerevisiae and several species using the onlineavailable ClustalW2 multiple sequence alignment tool, provided by EMBL-EBI. Identical residues are marked with (|) ; similarities (:) and totally different ones with (.). Residues included in the mutational analysis are highlighted. Three regions ( $a$, b and c) of sequence homology are indicated: (A) representing the mostly conserved part of the regulatory loop, (B) the conserved metal binding site, and (C) the largest homology domain on the c-terminus containing the active site. 
shows around $46 \%$ identity (Fig. 1) [14, 15]. This allows a large choice of conserved residues to be manipulated. Therefore, we employed data from structural and functional studies performed on Fbp1p from yeast and mammalian homologues to identify residues that are more likely to have a key role in catalysis and/or allosteric regulation.

A general overview of the alignment indicates a lack of homology in the $\mathrm{N}$ terminal region that extends for further 9 amino acids in the yeast Fbp1p compared to the mammalian homologues [15]. This part has been correlated to the glucose-mediated irreversible deactivation of yeast Fbp1p. The Pro1 residue has been implicated in targeting the enzyme to ubiquitin-mediated proteasome degradation in presence of glucose $[4,16]$. The residue Ser11 is linked to CAMP-mediated phosphorylation leading to loss of activity following glucose reintroduction to the medium [17]. Structural studies on porcine Fbp1p identified residues 52 to 72, as homologues to residues 63 to 83 in yeast, which form a loop that plays a crucial role in determining the tertiary structure of the tetramer, depending on the presence or absence of AMP and thus mediating AMP allosteric inhibition on the enzyme [18].

Two amino acids of interest in this loop are conserved between yeast and mammalian Fbp1p. The first is Asp68 in mammalian Fbp1p, Asp79 in the yeast homologue, which orients towards the active site, and facilitates the transfer of a proton from the phosphate group 1 of the substrate $\mathrm{F} 1,6 \mathrm{P} 2$ to the $\mathrm{OH}$ group of the product F6P. The second amino acid is the Asn64, Asn75 in yeast, which in addition to two other amino acids Asp 74 and Glu 98, Asp 85 and Glu 109 in yeast, contributes to the formation of a water- binding site. The water molecule bound to this site indirectly facilitates the nucleophilic attack of an adjacent water molecule on the phosphorus atom of the phosphate group on the position 1 of the substrate which consequently leads to the hydrolysis of the phosphate group and its splitting from the substrate [18].

The stride of residues Asp128 to Ser133 in yeast Fbp1p, or Asp118 to Ser 123 in mammalian Fbp1p, form an evolutionary conserved metal-binding motif. This sequence is largely conserved through pro and eukaryotic evolution; moreover it is encountered in various phosphatases that similarly require divalent cations as allosteric activators. In case of both mammalian and yeast Fbp1p the sequence is comprised of the following residues Asp-Pro-lle-Asp-GlySer [19]. Phe 194 is a conserved residue in both pro and eukaryotes, it lies within a conserved stretch of amino acids. Due to this, in addition to its high bulkiness, we presumed that this residue could be of significance for either the enzyme's folding or its protein/protein interactions.

The largest region of sequence homology in Fbp1p is on the carboxylic terminus and spans the residues Ala254 to Ser333 of the yeast homologue, and Ala243 to Ser321 of the mammalian enzyme, shows $66 \%$ sequence identity between the yeast and mammalian homologues. This region has been linked to the catalytic activity of the enzyme; a notion that has been deployed in recent studies on the human Fbp1p as well $[8,20]$. This c-terminal catalytic sequence contains two evolutionary conserved histidine residues; His265 and His324, both are very likely to comprise a part of the enzyme's catalytic domain.

TABLE 1. Summary of the analyzed mutations and utilized mutagenesis primers.

\begin{tabular}{|c|c|c|}
\hline $\begin{array}{l}\text { Mutations } \\
\text { (Altered residue) }\end{array}$ & Mutagenesis primers & $\begin{array}{l}\text { Abbreviation of the mutated } \\
\text { FBP1 form }\end{array}$ \\
\hline Pro1 $\rightarrow$ Ala1 & $\begin{array}{l}\text { fw: GGATTCTAGAACTAGTATGGCAAC TCTAGTAAA TGGACC } \\
\text { rev: GGTCCATTTACTAGAGTTGCCATACTAGTTCTAGAATCC }\end{array}$ & Pro1 \\
\hline Asn75 $\rightarrow$ Ala75 & $\begin{array}{l}\text { fw: GTTAGCAGGCGCTTCCGCCTTCACTGGTGACCAGC } \\
\text { rev: GCTGGTCACCAGTGAAGGCGGAAGCGCCTGCTAAC }\end{array}$ & Asn75 \\
\hline Asp79 $\rightarrow$ Ala79 & $\begin{array}{l}\text { fw: GCTTCCAACTTCACTGGTGCCCAGCAAAAGAAGTTGGAC } \\
\text { rev: GTCCAACTTCTTTTGCTGGGCACCAGTGAAGTTGGAAGC }\end{array}$ & Asp79 \\
\hline Glu109 $\rightarrow$ Ala109 & $\begin{array}{l}\text { fw: GGTCCTTGTATCTGAAGCACAGGAAGATTTGATCG } \\
\text { rev: CGATCAAGTCTTCCTGTGCTTCAGATACAAGGACC }\end{array}$ & Glu109 \\
\hline Asp131 $\rightarrow$ Ala131 & $\begin{array}{l}\text { fw: GTGTGTTGTGATCCTATTGCTGGCTCCTCAAATTTGGAC } \\
\text { rev: GTCCAAATTTGAGGAGCCACGAATAGGATCACAACACAC }\end{array}$ & Asp131 \\
\hline Ser133 $\rightarrow$ Ala133 & $\begin{array}{l}\text { fw: GTGATCCTATTGATGGCGCCTCAAATTTGGACGCCGG } \\
\text { rev: CCGGCGTCCAAATTTGAGGCGCCATCAATAGGATCAC }\end{array}$ & Ser133 \\
\hline Phe194 $\rightarrow$ Ala194 & $\begin{array}{l}\text { fw: GGTGATGGAGTTGATGGGGCTACCTTAGACACAAACTG } \\
\text { rev: CAAGTTTGTGTCTAAGGTAGCCCCATCAACTCCATCACC }\end{array}$ & Phe194 \\
\hline His65 $\rightarrow$ Ala265 & $\begin{array}{l}\text { fw: CCATGGTTGCTGATGTTGCCAGGACGTTTCTTTACGG } \\
\text { rev: CCGTAAAGAAACGTCCTGGCAACATCAGCAACCATGG }\end{array}$ & His265 \\
\hline His324 $\rightarrow$ Ala324 & $\begin{array}{l}\text { fw: GATTTGGTGCCAAGTCATACCGCTGACAAATCTTCTATT } \\
\text { rev: AATAGAAGATTTGTCAGCGATATGACTGGCACCAAATC }\end{array}$ & His325 \\
\hline
\end{tabular}


A

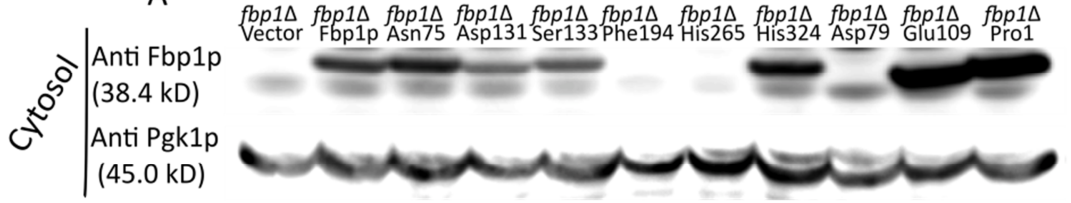

$$
\text { s| } \mid \begin{aligned}
& \text { Anti Fbp1p } \\
& (38.4 \text { kD) } \\
& \text { Anti Nop1p } \\
& (34.5 \text { kD) }
\end{aligned}
$$

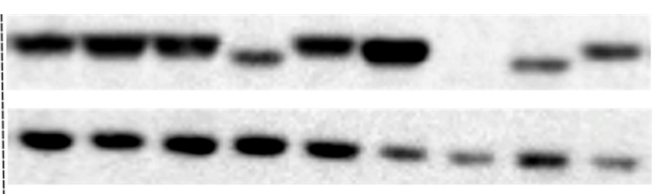

B

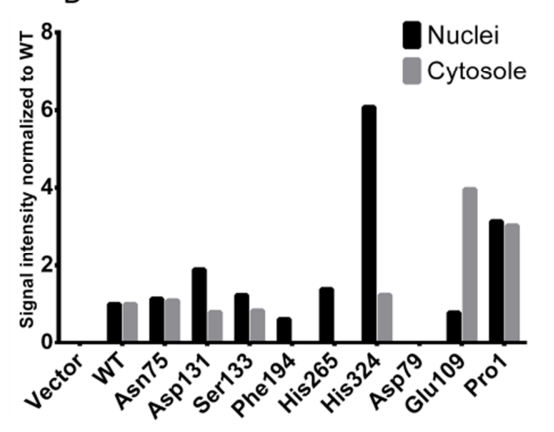

FIGURE 2: Immuno-detection of the over-expressed Fbp1p mutants. (A) Western-blot showing the levels of the over-expressed mutants in both cytosolic and nuclear fractions. Over-expression was verified in absence of endogenous Fbp1p expression in $f b p 1 \Delta$ strains. PGK1 and NOP1 were utilized as cytosolic and nuclear loading controls, respectively. (B) Quantification of the western-blot signals, each band was normalized to the intensity of its loading control, resulting values where then normalized to those of the wt.

Based on the aforementioned data, we screened 9 different mutated versions of yeast Fbp1p each having one of the formerly presented key-residues substituted with an alanine residue through a point mutation, alanine mutagenesis screening, as shown in Table 1.

In the following parts of this manuscript, mutated Fbp1p forms will be referred to using the number and three-letter symbol of the residue that had been replaced with alanine, these abbreviations are listed in Table 1.

\section{Overexpression levels of Fbp1p mutants}

Using immuno-blotting, 6 out of the 9 mutated forms of Fbp1p were successfully detected in the cytosolic fraction of the $f b p 1 \Delta$ yeast strains in which the mutated Fbp1p forms were over-expressed (Fig. 2A). No cytosolic bands



FIGURE 3: Enzymatic activity of the utilized FBP1 mutants. FBPase catalytic activity was measured in native lysates of wt BY4741 strains with over-expression of wt and mutated fbp1 cassettes. Measurements were performed in triplets, and error bars represent SD. Statistical significance was determined using student t-test for each of the mutants compared to both cells with control vector and those with over-expression of the wt Fbp1p. $\left({ }^{*}\right)$ Indicates statistically relevant difference compared to empty vector and $\left({ }^{\dagger}\right)$ compared to the wt Fbp1p. were detected for the Asp79, Phe194 and His 265 mutants. More mutants were successfully detected in nuclear fraction lysates of the different strains with mutated Fbp1p, with only the Asp79 mutant remaining undetected (Fig. 2A). Quantification of band intensities showed that the Asp131, Ser133 and His324 mutants were highly enriched in the nucleus compared to the wild-type (wt) form, and, interestingly, Phe194 and His265 mutants were fully retained in the nucleus (Fig. 2B).

\section{Catalytic activity of the Fbp1p mutants}

In order to screen for the impact of amino-acid substitutions on the catalytic activity of Fbp1p, we over-expressed the mutated cassettes of $f b p 1$ as well as wt $f b p 1$, in BY4741 wt haploid yeast strain. Subsequently we measured the fructose 1,6 bis-phosphatase (FBPase) enzymatic activity in the native protein lysates of the strains over-expressing the mutated forms of Fbp1p and statistically compared them to both cells over-expressing wt Fbp1p and those containing the control vector.

The assay shows that the mutants roughly cluster into 3 groups in respect of their exhibited enzymatic activity. Pro1 and Asn75 showed close-to-wild-type, only slightly reduced activity, nevertheless significantly different; Phe194, His265 and His324 diminished the activity to around 50\% compared to the wt; while Asp79, Glu109, Asp131 and Ser133 completely abrogated the activity stemming from over-expression showing a similar activity to extracts of cells transformed with empty vector (Fig. 3).

\section{Rescue of growth on non-fermentable medium (Drop-test on SDEG)}

In order to assess the mutants' potential of rescuing the gluconeogenic pathway in cells lacking FBP1 expression. The mutant $F B P 1$ forms were introduced into the $F B P 1$ knockout strain BY4741- $\triangle f b p 1$. Drop tests on SDEGmedium, containing glycerol and ethanol as exclusive carbon sources, were then performed with the fbp1 $\Delta$ strains over-expressing the Fbp1p mutant (Fig. 4A). Since the enzymatic activity of Fbp1p is indispensable for gluconeogenesis, the determining factor for the growth of each strain 
on the non-fermentable SDEG medium is the catalytic activity of the Fbp1p mutant expressed.

Four mutants, Asp79, Glu109, Asp131 and Ser133, completely failed to rescue growth on SDEG. The other screened mutants, Pro1, Asn75, Phe194, His265 and His 324 , clearly rescued growth on SDEG-agar exhibiting various levels of growth (Fig. 4A).

\section{Sensitivity to low doses of MMS}

Since we had shown previously that exposure to low doses of MMS triggers fbp1 transcription with the toxic outcome strongly depending on the presence of endogenous Fbp1p, we analyzed the effects of mutations in key residues of
Fbp1p on its ability to mediate increased sensitivity to MMS. For this purpose, expression vectors with mutated fbp1 cassettes were introduced into both wt and Fbp1pdeficient $f b p 1 \Delta$ BY4741 cells. Subsequently, the resulting strains were spotted on SD-Ura agar containing MMS concentrations ranging from $0.01 \%$ to $0.0175 \%$. These drop tests showed that the catalytically inactive mutants Asp79, Glu109, Asp131, Ser133 and His324 contributed no additional sensitivity towards MMS, and had growth levels comparable to wt cells harbouring control vector (Fig. 4B to I). Three catalytically active mutants Pro1, Phe194 and His265 conferred increased sensitivity toward MMS, when over-expressed in wt or Fbp1p-deficient $f b p 1 \Delta$ cells, these
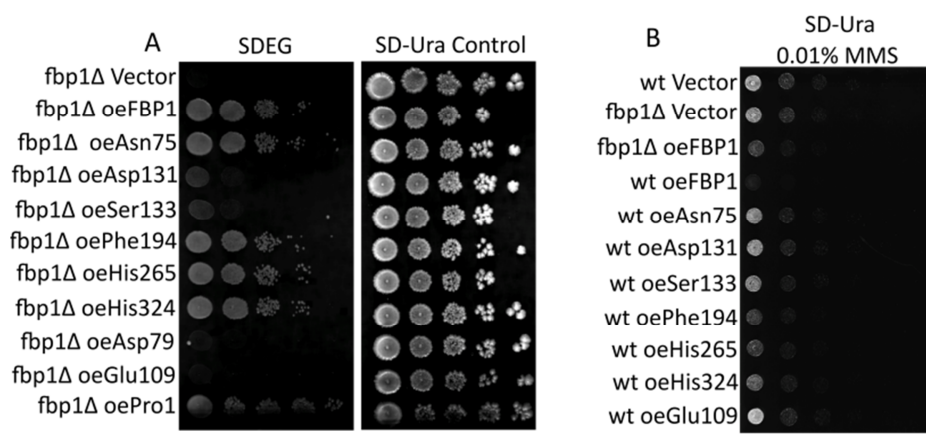
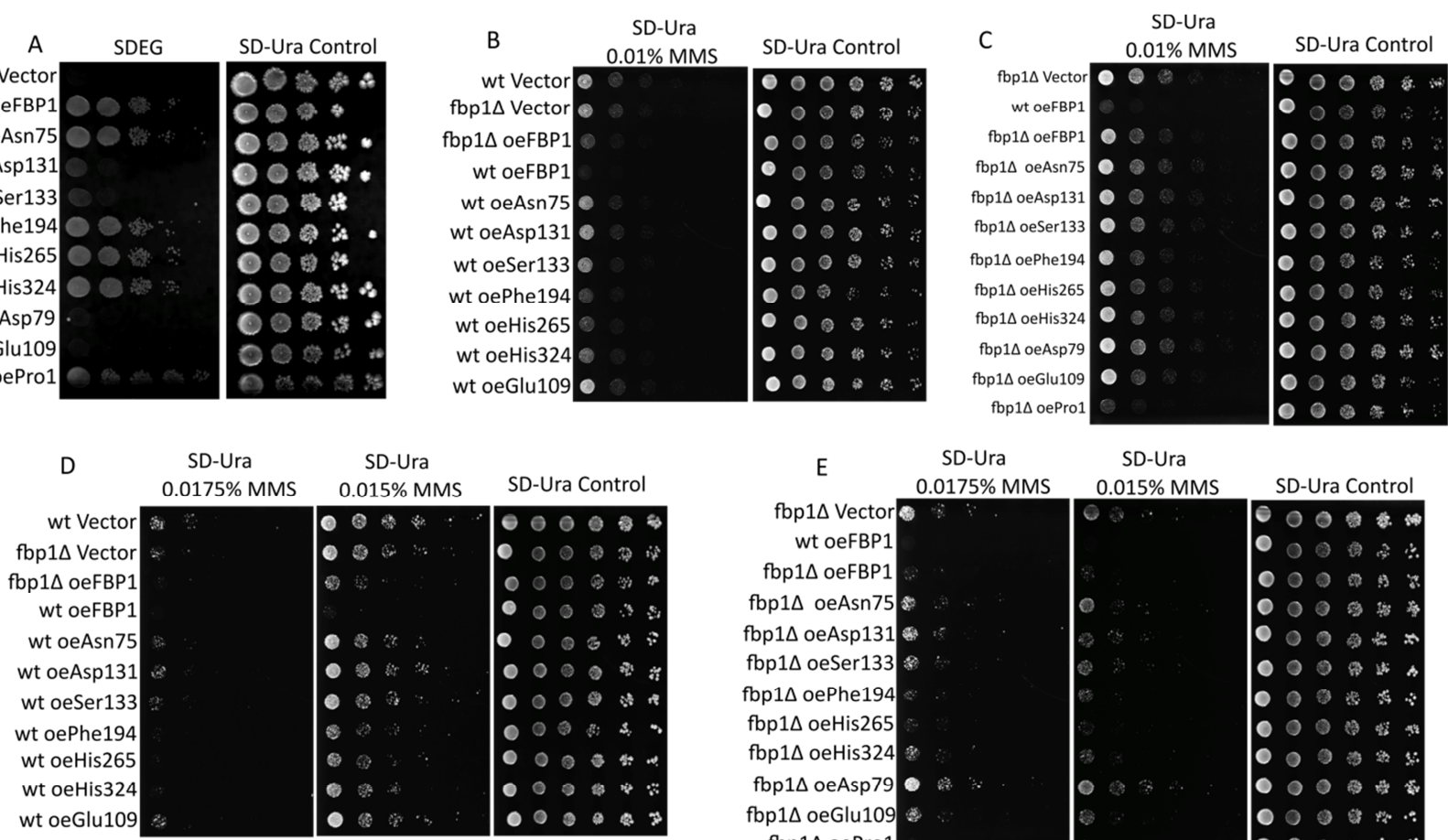

SD-Ura
$0.015 \% \mathrm{MMS}$
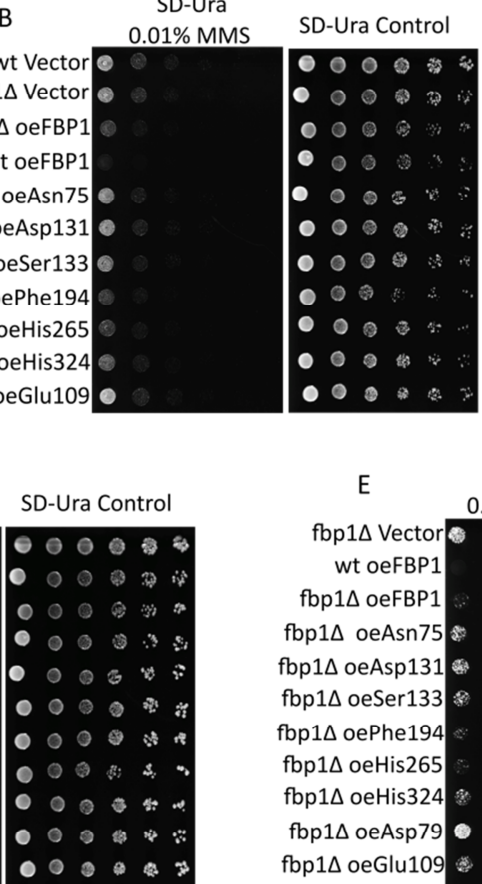

00 in:

SD-Ura



$000 \%$
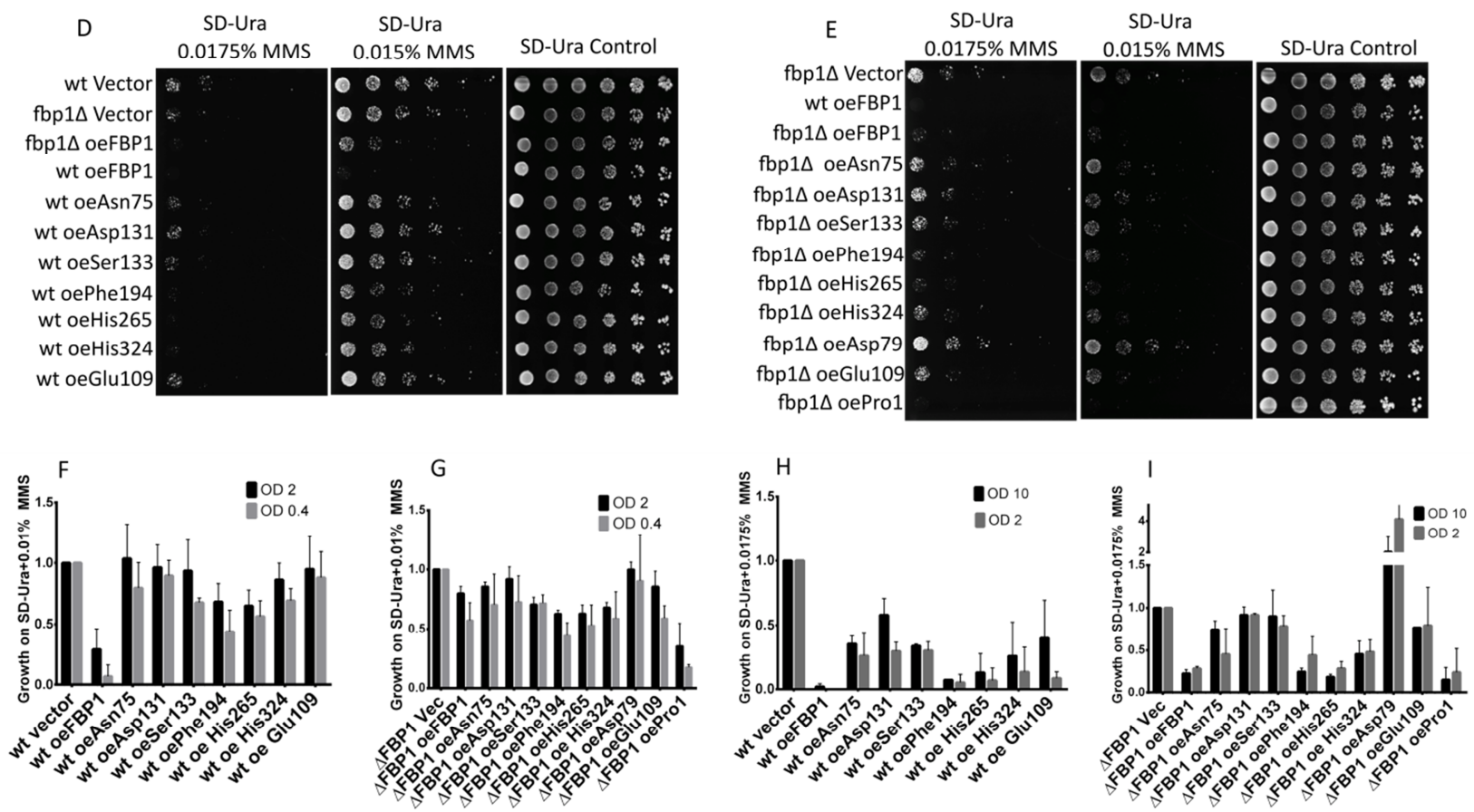

FIGURE 4: Drop-tests on Non-fermentable (SDEG) and MMS-containing agar-media. (A) Drop-test on SDEG non-fermentable medium. Wt and mutated $F B P 1$ cassettes were introduced into BY $4741 \mathrm{fbp} 1 \triangle$ cells lacking endogenous $F B P 1$, the resulting strains were grown in liquid SDUra medium, diluted and spotted on SDEG, same suspensions were spotted on SD-Ura agar as a control. Agar-plates were incubated for $48 \mathrm{~h}$ at $30^{\circ}$. (B and C) Drop tests on SD-Ura agar with $0.01 \%$ MMS. Mutants and wt cassettes were introduced into both wt (B) and $\triangle f b p 1$ (C) BY4741 cells. The resulting strains were grown in SD-Ura and then diluted (1:5) and spotted on freshly prepared SD-Ura containing $0.01 \%$, and on SD-Ura as non-treated control. Agar plates were incubated for $48 \mathrm{~h}$ at $30^{\circ} \mathrm{C}$. (D and E) Drop tests on SD-Ura agar containing $0.015 \%$ and $0.0175 \%$ MMS. Plates were incubated for $72 \mathrm{~h}$ at $30^{\circ} \mathrm{C}$. ( $\mathrm{F}$ to I) Quantifications of the spot-intensities of the drop tests on SD-Ura $0.01 \%$ MMS second and third spots $\left(\mathrm{OD}_{600}: 2\right.$ and 0.4 respectively) and $0.0175 \%$ first and second spots $\left(\mathrm{OD}_{600}: 10\right.$ and 2 respectively). Featured results are taken from three biologically independent replicates, (error-bars: SD; $\mathrm{N}=3$ ). 
A



E

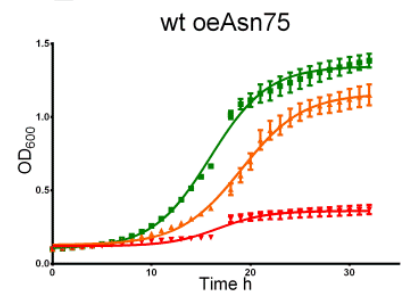

B

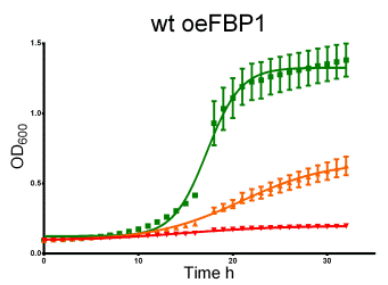

$\mathrm{F}$

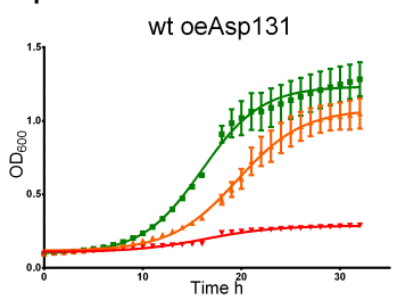

C

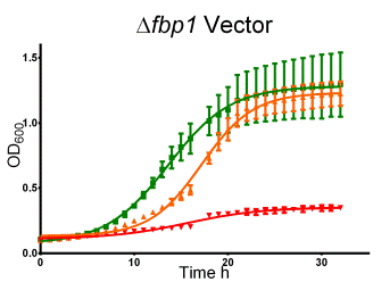

G

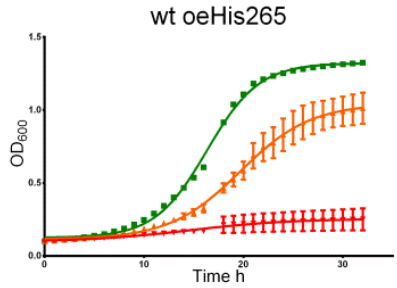

D

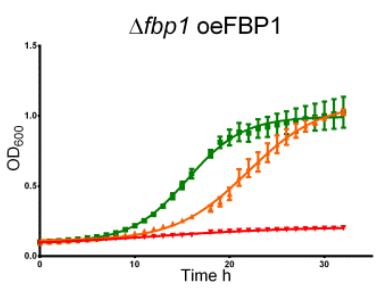

- NT

$=0.01 \% \mathrm{MMS}$

$0.02 \% \mathrm{MMS}$

FIGURE 5: Growth curves upon MMS treatment. Figures A to G feature the growth curves of the non-treated and MMS-treated (0.01\% and $0.02 \%$ ) yeast strains in liquid SD-Ura medium. Curves feature the increasing $\mathrm{OD}_{600}$ values over-time at $30^{\circ} \mathrm{C}$ with intermittent shaking, error bars represent SD, N = 3. (A) wt Vector, (B) wt oe FBP1, (C) fbp1 Vector, (D) fbp1 oe Fbp1p, (E) wt oeAsn75, (F) wt oe Ser133, (G) wt oe His265.

mutants impeded growth on MMS compared to the corresponding strains with control vector (Fig. $4 \mathrm{~B}$ to I). Interestingly, one catalytically active mutant, Asn75, contributed much less additional sensitivity towards MMS compared to wt and to other less catalytically active mutants (Fig. 2B to I). Similar trends were observed in both wt and $f b p 1 \Delta$ cells, with all wt-based strains showing less over-all growth with MMS concentrations of $0.01 \%$ and $0.0175 \%$. These results consist with our previously reported finding that $f b p 1$ deletion confers resistance towards low doses of MMS [13]. Quantifications of spot intensities of the drop-tests confirmed the eye-observable changes in MMS sensitivity with different Fbp1p mutations (Fig. 4F, G, H and I).

\section{Over-production of Fbp1p sensitises cells to MMS in liquid medium}

To better understand the cytotoxic effects of MMS, we also evaluated its impact on yeast proliferation in liquid media. Cultures were pre-incubated for $3 \mathrm{~h}$ and then treated with $0.01 \%$ and $0.02 \%$ MMS in 96 well plates, subsequently $\mathrm{OD}_{600}$ was measured over-time using a plate reader. While treatment with $0.02 \% \mathrm{MMS}$ resulted in severe inhibition of growth of all tested strains, the impact of $0.01 \%$ on proliferation in SD-Ura was clearly influenced by Fbp1p activity (Fig $5 A$ to $G$ ). Over-expression of Fbp1p generally reduced growth, however a clear contribution of the endogenous Fbp1p was also observed. Over-expression of Fbp1p in wt cells sensitized them to the lower MMS concentration of $0.01 \%$ leading to less over-all growth (Fig. 5A and B). In contrast, the effect of Fbp1p over-expression in cells lacking endogenous Fbp1p was very mild and limited to a temporary inhibition of growth (Fig. $5 \mathrm{C}$ and D). Three mutants were tested in comparison to over-expression of wt Fbp1p in the wt cell background. Over-expression of the catalyti- cally active mutants Asn75 and His265 led to a sensitizing effect at $0.01 \% \mathrm{MMS}$ similar to the effect seen with the wt Fbp1p (Fig. 5E and G), while the inactive mutant Asp131 had no noticeable effect (Fig. 5F).

The effect of Fbp1p over-production on cell survival depends on its genomic context of fbp1

We then sought to assess the impact of over-expression of Fbp1p or its mutants on cell survival upon MMS treatment using flow cytometry (FACS) analysis of PI-stained cells. After $12 \mathrm{~h}$ of MMS treatment in liquid SD-Ura cultures, all strains had a clear and significant increase in the amount of PI positive cells. Over-expression of wt Fbp1p in wt cells possessing endogenous Fbp1p led to a significant increase in the PI positive fraction upon MMS treatment with both $0.02 \%$ and $0.03 \%$ concentrations (Fig. $6 \mathrm{~A}$ ), whereas no significant increase in the PI-positive fraction was observed upon over-expression of Fbp1p in cells lacking endogenous Fbp1p (Fig. 6B). We also evaluated the effects of two catalytically active mutants, His265 and Asn75, and the totally inactive mutant Asp131 both in wt and $f b p 1 \Delta$ cells. Interestingly, non-of the tested mutants led to any significant difference in the PI-positive fraction after $12 \mathrm{~h}$ of MMS treatment (Fig. 6A and B).

Please note, that over-expression of Fbp1p was associated with a significant decrease in the PI-positive fractions of non-treated cells, which was also observed with tested mutants (Fig. 6A and B).

\section{Over-production of Fbp1p hampers colony-forming capac- ity upon MMS treatment}

To further investigate cell survival upon MMS treatment, we tested the impact of wt and mutated forms of Fbp1p on the colony forming capacity of cells following MMS treat- 

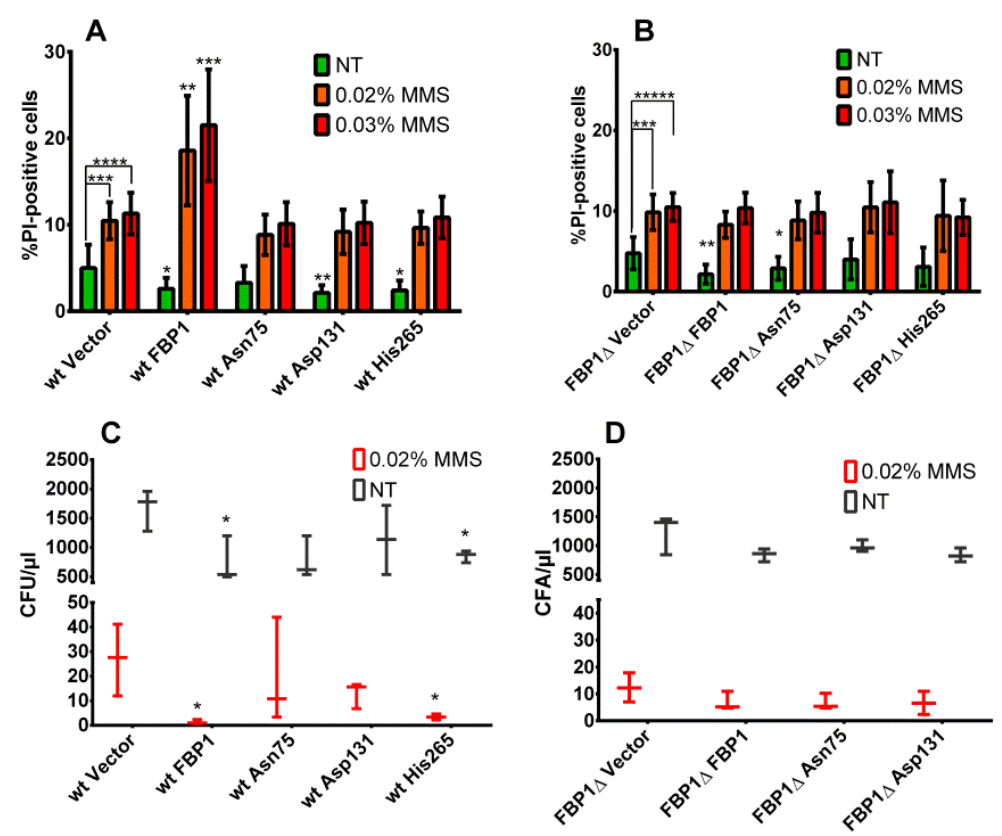

FIGURE 6: Cell survival and colony formation upon MMS treatment. (A and B) FACS analysis of PI staining following $12 \mathrm{~h}$ of MMS treatment. Bars represent the PI-positive percentage fractions of cells as measured using FACS. Results feature three biologically independent replicates with three technical replicates each. Error bars show the standard deviation. Statistical significance was determined using unpaired student $\mathrm{t}$-test assuming equal variance. $(*$ : $\mathrm{p}<0.05$; $^{* *}: \mathrm{p}<0.01 ;{ }^{* * *}: \mathrm{p}<0.001 ;{ }^{* * * *}: \mathrm{p}<$ 0.0001). The brackets over the wt Vector and fbp1s Vector strains the control vector strains show the significance comparing treated and non-treated of the same strain. While significance stars over the mutants represent significance when comparing the results form these strains with those of the control vector. (C and D) colony forming on SD-Ura agar following $12 \mathrm{~h}$ of MMS treatment in liquid SD-Ura. Results are from 3 independent experiments. Error bars show the standard deviation. Asterisks indicate statistically significant differences compared to the control vector strains. Significance was assessed using student t-test $(*: p<0.05)$. ment. Generally, $12 \mathrm{~h}$ of $0.02 \%$ MMS treatment lead to a two to three orders of magnitude decrease of the colony forming units $\mathrm{CFU} / \mu \mathrm{l}$ (Fig. $6 \mathrm{C}$ and D). In context of wt cells with endogenous Fbp1p expression, this decrease was strongest when wt Fbp1p or the active mutant His265 were over-expressed (Fig. 6C). In contrast, over-expression of wt Fbp1p in cells lacking the endogenous gene (fbp1 $1 \Delta)$ had no further inhibitory effect on colony forming capacity (Fig. 6D). It is also worth noting that in the wt strain, overexpression of Fbp1p or its active mutant His265 lead to a significant decrease in $\mathrm{CFU} / \mu \mathrm{l}$ in the non-treated samples as well, compared to non-treated cells harbouring the control vector.

\section{DISCUSSION}

This work aimed at exploring possible key evolutionaryconserved residues in Fbp1p linked to its apparent multiple-roles in both gluconeogenesis and response to MMS toxicity. In this study, we managed to identify several key residues essential for the enzymatic activity and the role in response to MMS-inflicted damage. The full activity exhibited by the Pro1 mutant completely fits with its reported role as a ubiquitination-mediating residue [16]. Replacing Pro1 should abrogate ubiquitination and lead to increased stability, which is clearly reflected in the increased intensity of the western-blot band of this mutant [4]. Additionally, when ectopically expressed in $f b p 1 \Delta$, the Pro1 mutant showed the highest MMS-sensitisation in the drop tests, even higher than wt Fbp1p, which could be explained by the higher stability of this mutant through evasion of proteasomal degradation. Replacing the residue Glu109 completely abolished the enzymatic activity, along with the ability to grow on non-fermentable carbon sources, however the Asn75 mutant almost maintained full activity compared to the wt, and also rescued growth on SDEG medium. This suggests that Glu109 is significantly participating in binding the catalytically essential water molecule while Asn75 seems to lack an important role in the formation of the water-binding pocket. The complete lack of enzymatic activity when Asp131 or Ser133 are replaced grants validity to their structurally presumed participation in the divalent-cation binding pocket and to the importance of the divalent cation for the activity of this enzyme.

Substituting Phe194 leads to a partial loss in enzymatic activity. This could be due to a direct influence on the catalytic activity. However, this lack of activity could also result from a change in molecular interaction and cellular localization, which was predominantly nuclear for this mutant.

Histidine residues in proximity to active sites on enzymes are usually presumed to contribute to the catalysis due to the amphiphilic nature of their side-chain imidazole ring [32]. In our study, altering either His265 or His324 reduced the FBPase catalytic activity by more than one fold; this supports the importance of these residues in facilitating the catalysis at the active site near the c-terminus.

Nevertheless, the activity is not entirely dependent on these residues. In both cases, the enzyme retains sufficient activity, and mediates the ability of growing on SDEG medium in transformed strains.

For most mutants, the observed sensitivity to MMS correlated to the measured FBPase catalytic activity, and the capacity to grow on SDEG non-fermentable medium. However, two mutants clearly defied this pattern. Asn75 showed full enzymatic activity, and also permitted growth on SDEG medium. However, Asn75 showed much less inhibition of growth on SD-Ura with MMS compared to the wt enzyme and other catalytically active mutants. This effect was verified repeatedly in drop-tests using both wt and fbp1 $1 \Delta$ strains. Similarly, the His324 mutant, exhibiting al- 
A


B

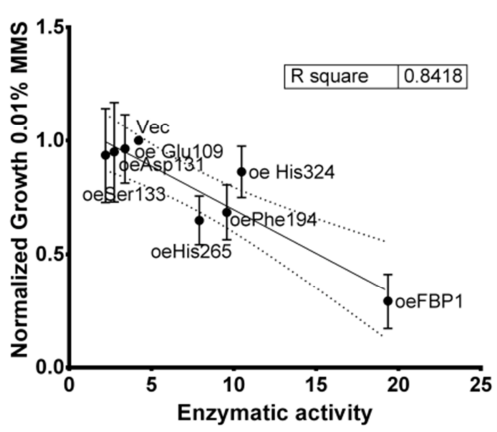

D

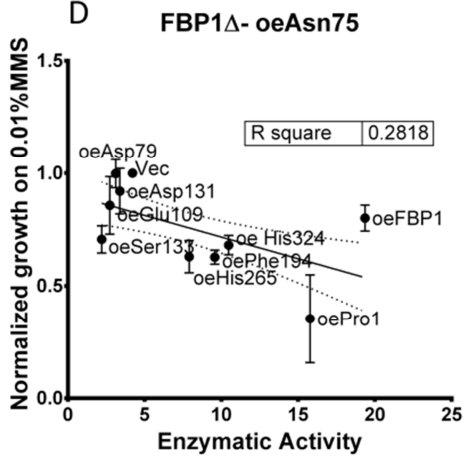

FIGURE 7: Linear correlation between enzymatic activity and growth on SD-Ura agar with $0.01 \%$ MMS in drop-tests. (A) wt strains; (B) wt strains excluding Asn75; (C) $f b p 1 \Delta$ strains; (D) $f b p 1 \Delta$ strains excluding Asn75. MMS growth values are taken from the spot-intensity quantification of three independent drop tests on SD-Ura agar with $0.01 \%$ MMS. $\mathrm{N}=$ 3; error bars represent SD. most $50 \%$ of the original catalytic activity, did not significantly increase sensitivity towards MMS in the fbp1 strain. The decoupling of MMS-sensitisation from the FBPase activity in case of the Asn75 is further highlighted by investigating the linear correlation between the measured FBPase activity of the mutants and their quantified growth on $0.01 \% \mathrm{MMS}$ in drop-tests (Fig. 7). In case of wt cells with intact endogenous Fbp1p, Asn75 lies far from the correlation line out of the confidence interval, showing less negative correlation between its catalytic activity and the growth of its hosting strain on MMS in drop-tests (Fig. 7A). Furthermore, the exclusion of the Asn 75 from the analysis, leads to substantial improvement in the linear correlation marked by an increase of R-square value from $\approx 0.37$ to $\approx 0.84$ (Fig 7A and B). Thus, the Asn75 and also possibly the His324 decouple the catalytic activity of the enzyme from its MMS-sensitizing role.

Substituting the residue His265 reduced enzymatic activity to less than half of wt Fbp1p, whereas this mutant conveyed even higher sensitivity towards MMS compared to the wt enzyme. In case of this His265 mutant it must be noted that it was enriched in the nuclear fraction and could not be detected in cytosolic extracts by western-blot, which may lead to lower activity in the enzymatic activity assay and may contribute to its increased MMS-sensitizing effect.

Taken together, our observations that for some mutants the catalytic activity of Fbp1p is decoupled from its additional role mediating sensitivity to MMS treatment suggest that these effects are mediated by two at least partially independent sites in the enzyme, one for enzymatic activity and one underlying the MMS-response. This clearly contradicts the alternative model, in which the
MMS-sensitizing role of $F B P 1$ would be directly linked to its catalytic activity and its role in gluconeogenesis.

Hypothesising on more speculative explanations that potentially could clarify the role of $F B P 1$ in response to MMS treatment, we searched for previously reported interactions of FBP1 in the literature. A physical interaction with TORC1 was reported in studies analyzing Fbp1p degradation upon glucose reintroduction following long-term glucose starvation [21, 22]. High throughput screenings also showed a genetic interaction with TOR2 [23]. TORC1 is a nutrient-sensing protein-kinase complex that responds to nutrient abundance by driving anabolic pathways required for cell growth and proliferation in addition to promoting cell cycle progression [24, 25]. Under glucose starvation, Fbp1p binds to TORC1, with the latter being essential for glucose-mediated Fbp1p degradation [21, 22]. These findings suggest a potential scenario in which the Fbp1p expressed in response to MMS treatment also binds to and inhibits TORC1 leading to a more significant inhibition of cell proliferation. Such Fbp1p-mediated loss of TORC1 function would consist with the increased viability upon MMS treatment in absence of Fbp1p, it could also explain the observed uncoupling of the enzymatic activity from MMS-sensitization. Other reported genetic interactions suggest an involvement of FBP1 in cell cycle regulation, these include: $\mathrm{CDH} 1$ [23], a cell-cycle regulated mitotic exit protein, GDH1 [26] glycogen phosphorylase postulated to co-ordinate metabolism to cell-cycle, TAF1 [27] involved in G1/S progression of cell cycle and ADK1 [26], involved in purine metabolism and response to DNA-replication stress. The aforementioned interactions make it very intriguing to functionally probe these suggested interactions and their effects on cell cycle progression in context of DNA damage, 


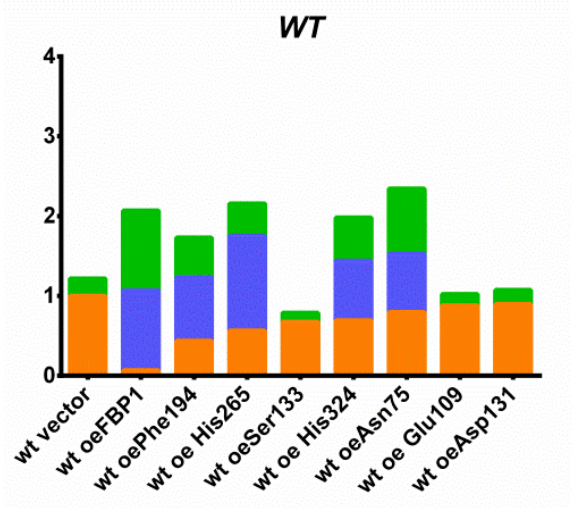

Enzymatic activity (u/mg total protein)

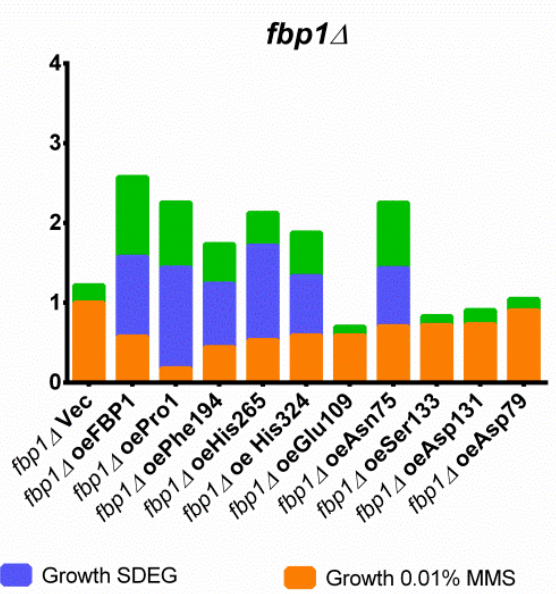

FIGURE 8: Summary figure. This figure summarizes the observed effects of the investigated mutations on FBPase catalysis, growth on non-fermentable SDEG and growth on SD-Ura agar containing 0.01\% MMS. Featured are the normalized values corresponding to enzymatic activity measured in the wt-strain background, image quantification of drop-tests on SDEG of the fbp $1 \Delta$-derived strains and averages of three independent replicates of drop-tests on SD-Ura agar with $0.01 \%$ MMS with both wt and $f b p 1 \Delta$ strains. Enzymatic activity and growth on SDEG were normalized to the strain over-expressing wt Fbp1p, while growth on SD-Ura agar with 0.01\% MMS was normalized to the strain harbouring empty vector.

an interesting open question we intend to pursue in the near future.

Our findings consistently showed that the outcome of FBP1 over-expression is very dependent on its genetic context and the presence of endogenously expressed Fbp1p. This is in line with earlier reports that MMS treatment induces the transcription of FBP1 within the first hour of treatment [13]. It is also already known that the lack of fbp1 on a genomic level confers more resistance against MMS treatment [13]. In this work we found that the impact of the ectopically expressed Fbp1p on cell survival upon MMS treatment largely depends on the presence of the endogenously expressed enzyme. Whereas overexpression of Fbp1p inhibits growth on SD-Agar MMS in both wt and $f b p 1 \Delta$ genotypes, the presence of endogenous Fbp1p seems to be the decisive factor when it comes to the impact of ectopically expressed Fbp1p on cell survival and sustained damage upon MMS treatment, this is made clear by the drastically different outcomes of PI-staining and colony formation of MMS-treated cells with Fbp1p over-expression when using the wt or fbp1 $\Delta$ cells. Additionally our observations support the notion that $F B P 1$ delays aging in yeast as evident by the significant decrease in PI-positive fractions of non-treated cells with Fbp1p over-expression compared to the vector control.

A summarizing figure (Fig. 8) depicts the observed effects of the mutants on three studied phenomena: catalytic FBPase activity, growth on non-fermentable carbon sources (SDEG) and growth on SD-Ura agar with $0.01 \%$ MMS in drop tests. The bars in the graph are sorted by decreasing the MMS-sensitivity (increasing values of growth on MMS). The decoupling of MMS-sensitisation from catalytic activity, discussed above, is again clearly visible in this figure. While the Asn75 mutant, catalytically active and conferring growth on SDEG, does not significantly sensitize cells to MMS in both wt and $f b p 1 \Delta$ genetic backgrounds, in case of His324, which is also catalytically active and confers growth on SDEG, the decoupling is most clearly visible in the wt strain.
In this work we successfully identified several conserved key residues for the catalytic function and/or the increased sensitivity to MMS and provide a clear separation of both activities. Our observation in yeast fits well with a recent report on FBP1 protein interactions in human tumours, which also seem to be independent of its catalytic activity $[8,28]$.

\section{MATERIAL AND METHODS}

\section{Utilized yeast strains, media and growth conditions}

All yeast strains were cultured and utilized in their haploidic-a form. Strains with the genetic background BY4741 (either wt or $\Delta f b p 1)$ were used to over-express the mutated versions of Fbp1p and screen for the effects of the mutations on enzymatic activity, growth on non-fermentable SDEG medium and sensitivity towards MMS treatment.

Transformed strains were maintained on synthetic minimal medium without uracil (SD-Ura) agar, containing $6.7 \mathrm{~g} / \mathrm{l}$ yeast nitrogen base, $20 \mathrm{~g} / \mathrm{l}$ glucose $100 \mathrm{mg} / \mathrm{l}$ hestidine, lucien and lysine. Prior to treatments starting cultures were made by inoculating a single colony into $2 \mathrm{ml}$ of SD-Ura liquid medium and incubating at $30^{\circ} \mathrm{C}$ with shaking overnight.

\section{Plasmid vectors}

Wt and mutated cassettes of FBP1 were over-expressed under the yeast GPD promoter using the high-copy number pRS426 plasmid vector containing Ura3 as a selection marker in yeast.

\section{Preparation of $f b p 1$ mutant plasmids}

The plasmid pRS426fbp1containing the wt fbp1 cassette was obtained from previous work performed in our lab [13].

Mutants of $f b p 1$ were prepared using the PCR-based sitedirected mutagenesis method using primers containing the desired point mutations, in combination with a commercially available kit "QuickChange ${ }^{\circledR}$ XL Site-directed mutagenesis kit" (Stratagen). Mutagenesis-PCR reactions were carried out according to the kit's instructions and 9 mutants of $f b p 1$ were prepared, each containing a single point mutation leading to the conversion of the codon of the targeted residue into an alanine codon. 
The mutated residues, their corresponding mutagenesis primers and symbols used to designate them in this work are featured in table 1.

\section{Transformation of yeast cells}

Competent Yeast cells were prepared and transformed using the Lithium Acetate and heat shock assisted method described by Gietz et al. [29].

Afterwards, successfully transformed cells were selected and further cultured on SD-Ura3 medium.

\section{Protein extraction from yeast cells}

Cells from over-night culture in SD-Ura medium were pelleted at $1000 \times \mathrm{g}$. Afterwards pellets were re-suspended in $1 \mathrm{ml} \mathrm{0,8}$ $\mathrm{M} \mathrm{NaCl}$ containing $0,2 \mathrm{mg}$ Zymolase and incubated for $30 \mathrm{~min}$ at room temperature. The resulting spheroplasts were then spun down and re-suspended in $1 \mathrm{ml} 10 \mathrm{mM}$ Tris containing protease inhibitors. Subsequently ca. $100 \mu$ l glass beads were added and spheroplasted cells were crushed using the beadmill homogeniser MM300 (Retsch) at full speed for $10 \mathrm{~min}$ followed by $10 \mathrm{~min}$ on ice. This homogenization/cooling cycle was repeated three times and lysates were obtained.

Nuclei were isolated form the rest of the lysates using differential centrifugation at $1000 \times \mathrm{g}$ for $5 \mathrm{~min}$. The supernatant containing the cytosolic fraction of the lysate was isolated and stored at $-20^{\circ} \mathrm{C}$, and the yielded pellet with the nuclear fraction was resuspended in $0,5 \mathrm{ml} 10 \mathrm{mM}$ Tris then centrifuged at $300 \mathrm{x} g$ to remove the cell debris. The remaining supernatant after this step contains the floating nuclei and was isolated and stored at $-20^{\circ} \mathrm{C}$. Both nuclear and cytosolic fractions were analyzed using SDS-Page followed by western blotting to investigate the presence of the various mutated forms of Fbp1p in transformed strains containing their respective overexpression vectors.

\section{Immuno-blotting for detection of Fbp1p and its mutant forms}

Denatured cytosolic and nuclear protein extracts were resolved using SDS-Page with $10 \%$ poly-acrylamide gels. Separated proteins were subsequently transferred onto PVDF membranes by semi-dry blot. Following blocking in 5\% BSA at $4^{\circ} \mathrm{C}$ over-night, membranes were incubated with the primary polyclonal anti-FBP1 antibody in 5\% BSA solution for 1 hour at room temperature. Subsequently membranes were washed 3 times with TBS-tween and then incubated with a 1:10000 diluted solution of anti-rabbit secondary antibody. After 5 washing steps membranes were developed using the Chemiluminescence reagent Western-Lighting, which consists of the reagents oxidizing and enhanced luminol mixed in 1:1 ratio. After $1 \mathrm{~min}$ of developing the membranes the signal was visualized using Fuji LAS-3000 imaging system and the AIDA image analyzing software.

The utilized anti yeast-FBP1 antibody is directed against the internal region of the protein, and was granted to our lab as courtesy of the group of Prof. Dr. Dieter Wolf at University of Stuttgart.

\section{Enzymatic assay of FBPase catalytic activity}

Total fructose 1,6 bis-phosphatase activity was measured using cytosolic protein extracts of over-night cultures of yeast cells as described by Skalecki et al. (1995) [30]. The general principle of the assay depends on coupling FBPase catalysis to the formation of NADPH through the phospho-glucose isomerase PGI and glucose-6-phosphate dehydrogenase G6PDH present in the reaction mix. Dephosphorylation of fructose 1,6 bis-phosphate by Fbp1p yields Fructose 6 phosphate, which is then converted into glucose 6 phsophate by PGI. G6P is then oxidized by G6PDH with NADP+ getting reduced to NADPH. Thus the ratio of the yielded F6P molecules and the NADPH is $1: 1$. NADPH has an absorption peak at $340 \mathrm{~nm}$, this absorption is then kinetically measured every 2 min for 20 cycles and then the total FBPase catalytic activity is estimated by the increase in absorption at $340 \mathrm{~nm}$ over time, using the following equation:

$$
\text { FBPase }[m U / m g]=\frac{\left(O D_{30}^{\prime 2}[m U]-O D_{40}^{\prime 1}[m U]\right) \times 161}{(2-t 1) \times \operatorname{Protein}[m g / m g]}
$$

\section{Growth measurements in liquid cultures}

Over-night cultures were first diluted to an $\mathrm{OD}_{600}$ of 0.2 , and shaken for $3 \mathrm{~h}$. Subsequently cultures were transferred into 96 round-bottom well-plates, $200 \mu \mathrm{l}$ per well. Of each used cell type, three wells were treated with $0.02 \% \mathrm{MMS}$, and three wells with $0.01 \% \mathrm{MMS}$ while four wells were kept untreated. The 96 well plates were then incubated at $30^{\circ} \mathrm{C}$ inside the microplate reader (Tecan-Ultra). $\mathrm{OD}_{600}$ of each well was measured every hour for $32 \mathrm{~h}, 15$ min of shaking within the plate reader preceded each measurement.

\section{Drop-tests}

Growth on non-fermentable carbon sources, drop test on SDEG medium

Starting from Mid-Log phase cultures, cell suspensions with the following dilution factors were prepared via serial dilution (1:10).

\section{$1: 1 ; 1: 10 ; 1: 100 ; 1: 1000 ; 1: 10000 ; 1: 100000$}

Subsequently, $4 \mu$ l of each cell suspension of the examined yeast strains were adjacently dropped onto SDEG-agar. Agar plates were incubated for $48 \mathrm{~h}$ at $30^{\circ} \mathrm{C}$ and then imaged to assess the growth of the strains over-expressing either wt or mutant versions of Fbp1p.

\section{Sensitivity to MMS treatment}

Starting from over-night cultures, cell-suspensions OD was adjusted to 10 , and these suspensions were serially diluted (1:5) to yield suspensions with the following OD600 (10; 2; 0.4; $0.08 ; 0.0016 ; 0.00032) .4 \mu$ l of each suspension was then spotted onto freshly prepared SD-Ura plates containing varying concentrations of MMS (0.01\% to $0.0175 \%)$.

The plates with $0.01 \% \mathrm{MMS}$, and those with $0.015 \%$ or $0.0175 \% \mathrm{MMS}$ were incubated at $30^{\circ} \mathrm{C}$ for $48 \mathrm{~h}$ and $72 \mathrm{~h}$, respectively.

\section{Drop-test Image-Quantification}

Spot-intensities from drop-tests were quantified using ImageJ, spot intensities from MMS plates were then normalized to those of the non-treated control, and values from mutants were normalized against the strain with wt Fbp1p.

\section{Cell survival upon MMS treatment}

Quantification of unviable cell fractions and colony formation assay upon MMS treatment

Unviable cells were detected using Propidium lodide (PI). PI is a red-fluorescent dye that stably binds the DNA once it enters 
cells. While healthy cells retain PI out of their intact membranes, disrupted membranes of severely damaged and/or dead cells are permeable to PI. Therefore PI can be used to differentiate the unviable and severely damaged cells from the rest of the healthy cell population [31].

Over-night cultures in SD-Ura were first diluted to an $\mathrm{OD}_{600}$ of 0.2 and incubated for $5 \mathrm{~h}$ till it reached mid-log phase. Each culture was then split into three parts, non-treated and treated with $0.02 \%$ and $0.03 \%$ MMS. Following $12 \mathrm{~h}$ of shaking at $30^{\circ} \mathrm{C}$, cultures were diluted and cell density of all samples was adjusted to OD of 0.5. Subsequently the adjusted suspensions were used for both Pl-staining and colony formation assay. For PI-staining cell suspensions were pipetted in triplicates in a round-bottom 96 well-plate and then simultaneously stained with propedium-iodide at a concentration of 125 $\mu \mathrm{g} / \mathrm{ml}$ and shaken for $10 \mathrm{~min}$ in the dark. Afterwards, cells were analyzed using the automatic FACS-guava system. PIpositive sub-populations were determined and calculated as a percentage of the whole cell population. The experiment was repeated 3 times, each with three technical replicates.

For the colony formation assay, the adjusted cellsuspensions of non-treated samples were diluted 1:1000 (three step serial dilution $1: 10$ ) then $50 \mu$ of each diluted sample were spread on an SD-Ura agar plates. Adjusted suspensions of cells treated with $0.02 \%$ were diluted $1: 10$ and then $50 \mu \mathrm{l}$ of each diluted sample was spread on an SD-Ura agar plate.

\section{REFERENCES}

1. Pilkis SJ, Granner DK (1992). Molecular physiology of the regulation of hepatic gluconeogenesis and glycolysis. Annual review of physiology 54:885-909.

2. Warburg O (1930). Note on the metabolism of tumours. Biochem Z 228:257-258.

3. Diaz-Ruiz R, Rigoulet M, Devin A (2011). The Warburg and Crabtree effects: On the origin of cancer cell energy metabolism and of yeast glucose repression. Biochimica et biophysica acta 1807(6): 568-576.

4. Hammerle M, Bauer J, Rose M, Szallies A, Thumm M, Dusterhus S, Mecke D, Entian KD, Wolf DH (1998). Proteins of newly isolated mutants and the amino-terminal proline are essential for ubiquitinproteasome-catalyzed catabolite degradation of fructose-1,6bisphosphatase of Saccharomyces cerevisiae. The Journal of biological chemistry 273(39): 25000-25005.

5. Vander Heiden MG, Cantley LC, Thompson CB (2009). Understanding the Warburg effect: the metabolic requirements of cell proliferation. Science 324(5930): 1029-1033.

6. Zhu Y, Shi M, Chen H, Gu J, Zhang J, Shen B, Deng X, Xie J, Zhan X, Peng $C$ (2015). NPM1 activates metabolic changes by inhibiting FBP1 while promoting the tumorigenicity of pancreatic cancer cells. Oncotarget 6(25): 21443-21451.

7. Dong C, Yuan T, Wu Y, Wang Y, Fan TW, Miriyala S, Lin Y, Yao J, Shi J, Kang T, Lorkiewicz P, St Clair D, Hung MC, Evers BM, Zhou BP (2013). Loss of FBP1 by Snail-mediated repression provides metabolic advantages in basal-like breast cancer. Cancer cell 23(3): 316-331.

8. Li B, Qiu B, Lee DS, Walton ZE, Ochocki JD, Mathew LK, Mancuso A Gade TP, Keith B, Nissim I, Simon MC (2014). Fructose-1,6bisphosphatase opposes renal carcinoma progression. Nature 513(7517): 251-255.
Plates were incubated for $48 \mathrm{~h}$ at $30^{\circ}$ then colonies were manually scored.

This experiment was repeated three times independently.

\section{ACKNOWLEDGMENTS}

We acknowledge the financial support of the Deutsche Forschungsgemeinschaft and Ruprecht-Karls-Universität Heidelberg within the funding programme Open Access Publishing.

Ali Ghanem is supported by a doctoral fellowship from the German Academic Exchange Service DAAD.

\section{CONFLICT OF INTEREST}

The authors declare no conflict of interest.

\section{COPYRIGHT}

(C) 2017 Ghanem et al. This is an open-access article released under the terms of the Creative Commons Attribution (CC BY) license, which allows the unrestricted use, distribution, and reproduction in any medium, provided the original author and source are acknowledged.

Please cite this article as: Ali Ghanem, Ana Kitanovic, Jinda Holzwarth, Stefan Wölfl (2017). Mutational analysis of fructose1,6-bis-phosphatase FBP1 indicates partially independent functions in gluconeogenesis and sensitivity to genotoxic stress. Microbial Cell 4(2): 52-63. doi: 10.15698/mic2017.02.557

9. Conrad M, Schothorst J, Kankipati HN, Van Zeebroeck G, RubioTexeira M, Thevelein JM (2014). Nutrient sensing and signaling in the yeast Saccharomyces cerevisiae. FEMS Microbiol Rev 38(2): 254-299.

10. Lin SS, Manchester JK, Gordon JI (2001). Enhanced gluconeogenesis and increased energy storage as hallmarks of aging in Saccharomyces cerevisiae. The Journal of biological chemistry 276(38): 36000-36007. doi: 10.1074/jbc.M103509200

11. Simpson-Lavy KJ, Bronstein A, Kupiec M, Johnston M (2015). CrossTalk between Carbon Metabolism and the DNA Damage Response in S. cerevisiae. Cell Rep 12(11): 1865-1875.

12. Kitanovic A, Walther $\mathrm{T}$, Loret MO, Holzwarth J, Kitanovic I, Bonowski F, Van Bui N, Francois JM, Wolfl S (2009). Metabolic response to MMS-mediated DNA damage in Saccharomyces cerevisiae is dependent on the glucose concentration in the medium. FEMS yeast research 9(4): 535-551.

13. Kitanovic A, Wolfl S (2006). Fructose-1,6-bisphosphatase mediates cellular responses to DNA damage and aging in Saccharomyces cerevisiae. Mutation research 594(1-2): 135-147.

14. Entian KD, Vogel RF, Rose M, Hofmann L, Mecke D (1988). Isolation and primary structure of the gene encoding fructose-1,6bisphosphatase from Saccharomyces cerevisiae. FEBS letters 236(1): 195-200.

15. Rogers DT, Hiller E, Mitsock L, Orr E (1988). Characterization of the gene for fructose-1,6-bisphosphatase from Saccharomyces cerevisiae and Schizosaccharomyces pombe. Sequence, protein homology, and expression during growth on glucose. The Journal of biological chemistry 263(13): 6051-6057.

16. Schork SM, Thumm M, Wolf DH (1995). Catabolite inactivation of fructose-1,6-bisphosphatase of Saccharomyces cerevisiae. Degradation occurs via the ubiquitin pathway. The Journal of biological chemistry 270(44): 26446-26450. 
17. Rittenhouse J, Harrsch PB, Kim JN, Marcus F (1986). Amino acid sequence of the phosphorylation site of yeast (Saccharomyces cerevisiae) fructose-1,6-bisphosphatase. The Journal of biological chemistry 261(9): 3939-3943.

18. Choe JY, Fromm HJ, Honzatko RB (2000). Crystal structures of fructose 1,6-bisphosphatase: mechanism of catalysis and allosteric inhibition revealed in product complexes. Biochemistry 39(29): 85658574.

19. York JD, Ponder JW, Majerus PW (1995). Definition of a metaldependent/Li(+)-inhibited phosphomonoesterase protein family based upon a conserved three-dimensional core structure. Proceedings of the National Academy of Sciences of the United States of America 92(11): 5149-5153.

20. Xu GJ, Datta AG, Singh VN, Suda H, Pontremoli S, Horecker BL (1981). Rabbit liver fructose 1,6-bisphosphatase: labeling of the active and allosteric sites with pyridoxal 5-phosphate and sequence of a nonapeptide from the active site. Archives of biochemistry and biophysics 210(1): 98-103.

21. Yan Y, Kang B (2010). Regulation of Vid-dependent degradation of FBPase by TCO89, a component of TOR Complex 1. Int J Biol Sci 6(4): 361-370.

22. Brown CR, Hung GC, Dunton D, Chiang HL (2010). The TOR complex 1 is distributed in endosomes and in retrograde vesicles that form from the vacuole membrane and plays an important role in the vacuole import and degradation pathway. The Journal of biological chemistry 285(30): 23359-23370.

23. Costanzo M, Baryshnikova A, Bellay J, Kim Y, Spear ED, Sevier CS, Ding H, Koh JL, Toufighi K, Mostafavi S, Prinz J, St Onge RP, VanderSluis B, Makhnevych T, Vizeacoumar FJ, Alizadeh S, Bahr S, Brost RL, Chen Y, Cokol M, Deshpande R, Li Z, Lin ZY, Liang W, Marback M, Paw J, San Luis BJ, Shuteriqi E, Tong AH, van Dyk N, et al. (2010). The genetic landscape of a cell. Science 327(5964): 425-431.
24. Soulard A, Cohen A, Hall MN (2009). TOR signaling in invertebrates. Current opinion in cell biology 21(6): 825-836.

25. Moreno-Torres $M$, Jaquenoud $M$, De Virgilio C (2015). TORC1 controls G1-S cell cycle transition in yeast via Mpk1 and the greatwall kinase pathway. Nat Commun 6:8256.

26. Szappanos B, Kovacs K, Szamecz B, Honti F, Costanzo M, Baryshnikova A, Gelius-Dietrich G, Lercher MJ, Jelasity M, Myers CL, Andrews BJ, Boone C, Oliver SG, Pal C, Papp B (2011). An integrated approach to characterize genetic interaction networks in yeast metabolism. Nat Genet 43(7): 656-662.

27. van Pel DM, Stirling PC, Minaker SW, Sipahimalani $P$, Hieter $P$ (2013). Saccharomyces cerevisiae genetics predicts candidate therapeutic genetic interactions at the mammalian replication fork. G3 (Bethesda) 3(2): 273-282.

28. Phillips R (2014). Kidney cancer: FBP1 depletion feeds ccRCC. Nature reviews Urology 11(9): 482.

29. Gietz RD, Schiestl RH, Willems AR, Woods RA (1995). Studies on the transformation of intact yeast cells by the LiAc/SS-DNA/PEG procedure. Yeast 11(4): 355-360.

30. Skalecki K, Mularczyk W, Dzugaj A (1995). Kinetic properties of Dfructose-1,6-bisphosphate 1-phosphohydrolase isolated from human muscle. The Biochemical journal 310 ( Pt 3): 1029-1035.

31. Dengler WA, Schulte J, Berger DP, Mertelsmann R, Fiebig $\mathrm{HH}$ (1995). Development of a propidium iodide fluorescence assay for proliferation and cytotoxicity assays. Anti-cancer drugs 6(4): 522-532.

32. Markley JL (1975). Observation of histidine residues in proteins by nuclear magnetic resonance spectroscopy. Accounts of Chemical Research 8 (2): 70-80. 